\title{
Chaotic Diffusion on Periodic Orbits: The Perturbed Arnol'd Cat Map
}

\author{
Itzhack Dana and Vladislav E. Chernov \\ Minerva Center and Department of Physics, Bar-Ilan University, Ramat-Gan 52900, Israel
}

\begin{abstract}
Chaotic diffusion on periodic orbits (POs) is studied for the perturbed Arnol'd cat map on a cylinder, in a range of perturbation parameters corresponding to an extended structural-stability regime of the system on the torus. The diffusion coefficient is calculated using the following PO formulas: (a) The curvature expansion of the Ruelle zeta function. (b) The average of the PO winding-number squared, $w^{2}$, weighted by a stability factor. (c) The uniform (nonweighted) average of $w^{2}$. The results from formulas (a) and (b) agree very well with those obtained by standard methods, for all the perturbation parameters considered. Formula (c) gives reasonably accurate results for sufficiently small parameters corresponding also to cases of a considerably nonuniform hyperbolicity. This is due to uniformity sum rules satisfied by the PO Lyapunov eigenvalues at fixed $w$. These sum rules follow from general arguments and are supported by much numerical evidence.
\end{abstract}

PACS numbers: 05.45.Ac, 05.45.Mt, 45.05.+x

Typeset using REVTEX 


\section{INTRODUCTION}

Understanding to what extent chaotic motion in Hamiltonian systems exhibits random properties such as diffusion is a problem of both fundamental and practical importance. The existence of deterministic chaotic diffusion has been approximately established using a variety of approaches [1-20]. A systematic approach is based on the hierarchy of periodic orbits (POs) embedded in the chaotic region [10-20]. Let us summarize the main ideas of this approach by considering, for definiteness, the kicked-rotor maps on the cylinder

$$
l_{m+1}=l_{m}+f\left(x_{m}\right), \quad x_{m+1}=x_{m}+l_{m+1} \bmod 1,
$$

where $l$ is angular momentum, $x$ is angle, and the force function $f(x)$ satisfies $f(x+1)=f(x)$ and $f(-x)=-f(x)$. The diffusion coefficient for (1) is formally defined by:

$$
D=\lim _{m \rightarrow \infty} D_{\mathcal{E}}(m), \quad D_{\mathcal{E}}(m)=\frac{\left\langle\left(l_{m}-l_{0}\right)^{2}\right\rangle_{\mathcal{E}}}{2 m}
$$

where \langle\rangle$_{\mathcal{E}}$ denotes average over an ensemble $\mathcal{E}=\left\{\left(x_{0}, l_{0}\right)\right\}$ of initial conditions in a chaotic

region. Well known problems in a reliable numerical calculation of $D$ are: (a) The roundoff errors caused by the chaotic exponential instability. (b) The ambiguity in the choice of $\mathcal{E}$ and the iteration time $m$. These problems are systematically solved [10] by choosing $\mathcal{E}$ as the ensemble $\mathcal{U}_{n}$ of all the primitive POs of period $n$ in the chaotic region and $m=n$. For the map (1), a PO $p \in \mathcal{U}_{n}$ is generally defined by initial conditions $\left(x_{0}^{(p)}, l_{0}^{(p)}\right)$ satisfying

$$
l_{n}^{(p)}=l_{0}^{(p)}+w_{p}, \quad x_{n}^{(p)}=x_{0}^{(p)}
$$

where $n$ is the smallest integer for which (3) holds with integer $w_{p}$, the winding number of PO $p$. Since (1) is essentially periodic in $(x, l)$ with period $1,\left(x_{0}^{(p)}, l_{0}^{(p)}\right)$ can be restricted to a unit torus, $-0.5 \leq x_{0}^{(p)}, l_{0}^{(p)}<0.5$. The ensemble $\mathcal{U}_{n}$ may be viewed as an invariant "level $n$ " approximation of the chaotic region and the diffusion rate on $\mathcal{U}_{n}$ is given by [10]:

$$
D(n)=\frac{1}{2 n N(n)} \sum_{p \in \mathcal{U}_{n}} w_{p}^{2}=\frac{1}{2 n N(n)} \sum_{w} N_{w}(n) w^{2}
$$


where $N(n)$ is the total number of POs in $\mathcal{U}_{n}$ and $N_{w}(n)$ is the number of POs in $\mathcal{U}_{n}$ with $w_{p}=w$. Similarly, one can associate diffusion rates with subensembles of $\mathcal{U}_{n}$ having welldefined dynamical characteristics [10,11]. For uniformly hyperbolic systems, (4) is expected to approximate well the diffusion coefficient (2) associated with generic ensembles of aperiodic chaotic orbits. In fact, in the case of the cat and sawtooth maps [2], (4) gives the exact value of $D$ for the cat maps and approximates very well $D$ for the sawtooth maps [10].

The diffusion coefficient for generic chaotic ensembles in hyperbolic systems is given by the exact PO formula $[16,19,21]$ :

$$
D=-\left.\frac{1}{2} \frac{\partial^{2} \zeta^{-1}(\beta, s) / \partial \beta^{2}}{\partial \zeta^{-1}(\beta, s) / \partial s}\right|_{\beta=s=0} .
$$

Here $\zeta(\beta, s)$ is the Ruelle zeta function [21],

$$
\zeta^{-1}(\beta, s)=\prod_{p}\left[1-\exp \left(\beta w_{p}-s n_{p}\right)\left|\Lambda_{p}\right|^{-1}\right]
$$

where the product is over all the primitive POs, $n_{p}$ is the period of PO $p$, and $\Lambda_{p}$ is the associated Lyapunov eigenvalue $\left(\left|\Lambda_{p}\right|>1\right)$. One can express (6) as a power series in $\exp (-s)$ :

$$
\zeta^{-1}(\beta, s)=1+\sum_{n=1}^{\infty} c_{n}(\beta) \exp (-s n)
$$

where the $n>1$ terms are known as "curvatures" [19]. The convergence of (7) is generally better than that of (6). Formula (5) with a (truncated) curvature expansion (7) has been applied to several systems $[13,14,16-20]$. For uniformly hyperbolic maps with a complete symbolic dynamics (i.e., whose grammar is unrestricted by "pruning rules"), all the curvature terms in (7) vanish identically and the exact value of $D$ from (5) coincides precisely with the diffusion rate (4) for $n=1$. Examples of such trivial systems are $1 \mathrm{D}$ piecewise linear maps $[13,14]$ and chains of coupled baker maps [16,21]. The application of (5) and other PO formulas [15] to a more realistic system, the periodic Lorentz gas, gives results [18] that are within $8 \%$ of the values of $D$ obtained by standard methods. For standard maps (1), the quasilinear (strong-chaos) limit of $D$ is approximated by using just POs of period 
$n=1$ and $n=2$ [17]. For the cat and sawtooth maps, the quasilinear approximation of $D$ is reproduced by formulas related to (5) [20].

In this paper, chaotic diffusion on POs is studied for a nontrivial Hamiltonian system exhibiting a transition from uniform to nonuniform hyperbolicity as a parameter is varied. This is the perturbed Arnol'd cat map on the cylinder, defined by (1) with

$$
f(x)=f_{0}(x)+\frac{\kappa}{2 \pi} \sin (2 \pi x),
$$

where $f_{0}(x)=x$ for $|x|<0.5, f_{0}(-0.5) \equiv 0, f_{0}(x+1)=f_{0}(x)$, and $\kappa$ is a perturbation parameter. This system, with the definition $f_{0}(-0.5) \equiv-0.5$, is usually considered on a torus, $-0.5 \leq x, l<0.5$. Perturbed cat maps on the torus have attracted much attention recently in the context of "quantum chaos" [22-24]. Anosov theorem [25] states that the dynamics on the torus for sufficiently small $\kappa, \kappa<\kappa_{\mathrm{c}}$, is topologically equivalent to that of the unperturbed $(\kappa=0)$ system (in particular, the system is completely chaotic for $\kappa<\kappa_{\mathrm{c}}$ ). This expresses the well known structural stability of cat maps (see more details in Sec. II, where we determine $\left.\kappa_{\mathrm{c}} \approx 0.437\right)$. Actually, we provide numerical evidence in Sec. II that the structural-stability regime extends, at least approximately, beyond $\kappa_{\mathrm{c}}$, up to $\kappa \approx 1$. A fully chaotic regime is observed up to $\kappa \approx 1.5$. For larger perturbations, stability islands born by bifurcation, leading to a significant mixed phase space for $\kappa>1.7$ (see Fig. 1). We emphasize that unperturbed cat maps already feature a very nontrivial symbolic dynamics with nonexplicit pruning rules given by an infinite set of inequalities [26]. As a result, all the curvature terms in zeta-function expansions are nonvanishing. See, e.g., an exact expression of $\zeta^{-1}(0, s)$ for cat maps derived in Sec. II. The relevant dynamics on the cylinder can be easily inferred from that on the torus.

In Sec. III, we calculate accurately the diffusion coefficient $D$ for $\kappa$ up to $\kappa \approx 1$ using several PO formulas: (a) The curvature-expansion formula [(5) with (7)]. (b) The average of $w_{p}^{2}$ weighted by a stability factor. This formula was used in Refs. $[15,18]$ and we give 
a derivation of it from (5). (c) The nonweighted-average formula (4). The convergence of each formula as the order $n$ of approximation is increased is verified. When compared with results obtained by standard methods, the results from formulas (a) and (b) appear to be more accurate than those in previous works. The relative difference between the PO and standard results is not larger than $0.4 \%$ when formula (b) is used and not larger than $1.7 \%$ when formula (a) is used. Formula (c) gives reasonably accurate results (within $2 \%$ of the standard results) for sufficiently small values of $\kappa$ corresponding also to cases of a considerably nonuniform hyperbolicity. This is due to uniformity sum rules satisfied by the PO Lyapunov eigenvalues at fixed winding number $w$. These sum rules follow from general arguments and are supported by much numerical evidence.

\section{CAT MAPS AND STRUCTURAL STABILITY}

Consider the unit torus $\mathbb{T}^{2}:-0.5 \leq x, l<0.5$ and let $\mathbf{z} \equiv(x, l)$. Hyperbolic cat maps on $\mathbb{T}^{2}$ are defined by the map $\phi_{0}: \mathbf{z}^{\prime}=A \cdot \mathbf{z} \bmod \mathbb{T}^{2}$, where $A$ is a $2 \times 2$ integer matrix with $\operatorname{det}(A)=1$ and $\operatorname{Tr}(A)>2$. While these maps are uniformly hyperbolic, they feature a very nontrivial symbolic dynamics [26]. As a result, all the curvature terms in zeta-function expansions are nonvanishing. We show this here by deriving an exact expression of $\zeta^{-1}(0, s)$ for $\phi_{0}$. First, the uniform hyperbolicity implies that the Lyapunov eigenvalue $\Lambda_{p}$ of any PO with period $n$ is given by $\Lambda_{p}=\Lambda^{n}$, where $\Lambda$ is the largest eigenvalue of $A$. Then, from (6),

$$
\zeta^{-1}(0, s)=\prod_{n=1}^{\infty}\left[1-\rho^{n}(s)\right]^{N(n)}
$$

where $\rho(s)=\Lambda^{-1} \exp (-s)$ and $N(n)$ is the number of primitive POs of period $n$. For $\rho(s)<1$, we find from Rel. (9) that

$$
\begin{array}{r}
\ln \left[\zeta^{-1}(0, s)\right]=\sum_{j=1}^{\infty} N(j) \ln \left[1-\rho^{j}(s)\right]= \\
-\sum_{j=1}^{\infty} j N(j) \sum_{i=1}^{\infty} \frac{\rho^{i j}(s)}{i j}=-\sum_{n=1}^{\infty} \frac{\rho^{n}(s)}{n} \sum_{j \mid n} j N(j),
\end{array}
$$


where $j \mid n$ means that the positive integer $j$ divides $n$. We now use the general relation

$$
\sum_{j \mid n} j N(j)=P(n)
$$

where $P(n)$ is the number of periodic points of period $n$. For the cat maps one has [28]

$$
P(n)=\left|\operatorname{Tr}\left(A^{n}\right)-2\right|=\Lambda^{n}+\Lambda^{-n}-2 .
$$

Using (11) and (12) in (10), we obtain, for $s \geq 0$,

$$
\zeta^{-1}(0, s)=\exp \left[-\sum_{n=1}^{\infty} P(n) \frac{\rho^{n}(s)}{n}\right]=\frac{[1-\exp (-s)]\left[1-\Lambda^{-2} \exp (-s)\right]}{\left[1-\Lambda^{-1} \exp (-s)\right]^{2}} .
$$

The explicit curvature expansion of $\zeta^{-1}(0, s)$ for $s \geq 0$ is easily found from (13):

$$
\zeta^{-1}(0, s)=1-P(1) \sum_{n=1}^{\infty} n \Lambda^{-n} \exp (-s n) .
$$

Thus, all the curvatures $c_{n}(0), n>1$, are nonvanishing.

A perturbed cat map on $\mathbb{T}^{2}$ is given by $\phi_{\kappa}: \mathbf{z}^{\prime}=A \cdot \mathbf{z}+\kappa \mathbf{F}(\mathbf{z}) \bmod \mathbb{T}^{2}$, where $\mathbf{F}(\mathbf{z})$ is a smooth vector field periodic on $\mathbb{T}^{2}$. Anosov theorem [25] states that for sufficiently small $\kappa, \kappa<\kappa_{\mathrm{c}}, \phi_{\kappa}$ is topologically conjugate to $\phi_{0}$ by a continuous near-identity map $H_{\kappa}, \phi_{\kappa}=H_{\kappa} \circ \phi_{0} \circ H_{\kappa}^{-1}$. Thus, any orbit $\mathcal{O}_{\kappa}$ of $\phi_{\kappa}$, in particular a PO, can be written as $\mathcal{O}_{\kappa}=H_{\kappa} \mathcal{O}_{0}$, where $\mathcal{O}_{0}$ is some orbit of $\phi_{0}$. We emphasize that the relation $\phi_{\kappa}=H_{\kappa} \circ \phi_{0} \circ H_{\kappa}^{-1}$ does not imply that the Lyapunov eigenvalue $\Lambda_{p}$ of a PO $\mathcal{O}_{\kappa}=H_{\kappa} \mathcal{O}_{0}$ is equal to that of $\mathcal{O}_{0}$ since the map $H_{\kappa}$ is not differentiable [25]. The bound $\kappa_{\mathrm{c}}$ is generally determined by the inequality: $\max _{\mathbf{z} \in \mathbb{T}^{2}}(|\kappa \partial \mathbf{F} / \partial \mathbf{z} \cdot \mathbf{z}| /|\mathbf{z}|)<1-\Lambda^{-1}$, where $|\mathbf{z}|=\left(x^{2}+l^{2}\right)^{1 / 2}$. Choosing $A=(2,1 ; 1,1)$ (corresponding to the Arnol'd cat map [25]) and $\mathbf{F}(\mathbf{z})=(1 / 2 \pi) \sin (2 \pi x)(1,1)$, we see that $\phi_{\kappa}$ is just the map (1) with (8) and the definition $f_{0}(-0.5) \equiv-0.5$ [instead of $\left.f_{0}(-0.5) \equiv 0\right]$. In this case, $\Lambda=(3+\sqrt{5}) / 2$ and $|\partial \mathbf{F} / \partial \mathbf{z} \cdot \mathbf{z}|=\sqrt{2}|x| \cos (2 \pi x)$. From the inequality above we then get $\kappa<\kappa_{\mathrm{c}} \approx 0.437$.

The POs of a perturbed cat map for $\kappa<\kappa_{\mathrm{c}}$ can be calculated very accurately as follows. First, the POs $\mathcal{O}_{0}$ of the cat map are determined exactly using the techniques in Ref. 
[27]. The perturbed POs $\mathcal{O}_{\kappa}=H_{\kappa} \mathcal{O}_{0}$ are then computed by applying to $\mathcal{O}_{0}$ the map $H_{\kappa}$ constructed iteratively from the nonlinear functional equation satisfied by it, starting from the solution $H_{\kappa}^{(0)}$ of the "homological equation" (see details in the proof of Anosov theorem [25]). In this way, we have calculated all the POs $\mathcal{O}_{\kappa}=H_{\kappa} \mathcal{O}_{0}$ of the perturbed Arnol'd cat map with periods $n \leq 14$ with an accuracy of at least $10^{-10}$; this accuracy was checked by direct iteration of the map. As a matter of fact, we found no problems in extending these calculations beyond $\kappa_{\mathrm{c}} \approx 0.437$. We have thus verified that all the POs $\mathcal{O}_{\kappa}=H_{\kappa} \mathcal{O}_{0}$ with $n \leq 14$ exist at least up to $\kappa=0.946$. In order to check to what extent these POs cover the set of all POs with $n \leq 14$ for $\kappa_{\mathrm{c}}<\kappa \leq 0.946$, we have used the $\zeta^{-1}(0,0)=0$ test [16-19]: the curvature expansion (7) for $\zeta^{-1}(0,0)$, restricted to the set of POs $\mathcal{O}_{\kappa}=H_{\kappa} \mathcal{O}_{0}$, was calculated up to order $n=1, \ldots, 14$ for six values of $\kappa$ uniformly distributed in the interval $0<\kappa \leq 0.946$. The results are shown in Table I. We see that the behavior of $\zeta^{-1}(0,0)$ as $n$ increases is basically the same for both $\kappa>\kappa_{\mathrm{c}}$ and $\kappa<\kappa_{\mathrm{c}}$, and there is a general trend of $\left|\zeta^{-1}(0,0)\right|$ to decrease. This indicates that the POs $\mathcal{O}_{\kappa}=H_{\kappa} \mathcal{O}_{0}$ form, at least, a large fraction of all the POs for $\kappa_{\mathrm{c}}<\kappa \leq 0.946$, i.e., almost no bifurcations take place in this interval. Thus, the structural-stability regime appears to extend, at least approximately, up to $\kappa=0.946$. On the basis of this observation, only the POs $\mathcal{O}_{\kappa}=H_{\kappa} \mathcal{O}_{0}$ were used in our calculations for $0<\kappa \leq 0.946$.

On the cylinder, the system is described by the map (1) with (8) and $f_{0}(-0.5) \equiv 0$. The discontinuity of (8) at $x=-0.5$ can be viewed as an infinite-slope (vertical) segment. Is is then easy to show that POs with points on $x=-0.5$ must have an infinite value of $\Lambda_{p}$. Thus, they will not contribute to (6) and will not be considered. Clearly, the initial conditions for the relevant POs (having no point on $x=-0.5$ ) can be chosen as points of torus POs $\mathcal{O}_{\kappa}=H_{\kappa} \mathcal{O}_{0}$ lying in the domain $|x|,|l|<0.5$. The number $N(n)$ of relevant POs used in our diffusion calculations in Sec. III is listed in Table II. The winding number $w_{p}$ is calculated from (3). Strictly speaking, there is no structural stability on the cylinder since $w_{p}$ generally changes when $\kappa$ is varied in any interval, in particular $\left(0, \kappa_{\mathrm{c}}\right)$. 


\section{RESULTS FOR $D$ AND UNIFORMITY SUM RULES}

We start by deriving a general formula [Eq. (17) below] giving an approximation to $D$ in terms of all the primitive POs of period $n$. Let us calculate the derivatives $\partial^{2} \zeta^{-1}(\beta, s) / \partial \beta^{2}$ and $\partial \zeta^{-1}(\beta, s) / \partial s$ in (5) for $\beta=0$ and $s>0$ by direct differentiation of the infinite product (6), which is convergent and nonvanishing for $s>0[16,21]$. Because of the inversion symmetry of $(1)[f(-x)=-f(x)]$, for each $\mathrm{PO}$ with characteristics $\left(w_{p}, \Lambda_{p}\right)$ there exists a PO with characteristics $\left(-w_{p}, \Lambda_{p}\right)$. This implies that $\partial \zeta^{-1}(\beta, s) /\left.\partial \beta\right|_{\beta=0, s>0}=0$. Using this fact and taking the limit of $s \rightarrow 0^{+}$, we obtain the following expression for $D$ :

$$
D=\lim _{n \rightarrow \infty} \frac{\sum_{n^{\prime}=1}^{n} g\left(n^{\prime}\right) D_{\mathrm{WA}}\left(n^{\prime}\right)}{\sum_{n^{\prime}=1}^{n} g\left(n^{\prime}\right)},
$$

where

$$
\begin{gathered}
g(n)=n \sum_{p \in \mathcal{U}_{n}} \frac{\left|\Lambda_{p}\right|^{-1}}{1-\left|\Lambda_{p}\right|^{-1}}, \\
D_{\mathrm{WA}}(n)=\frac{1}{2 g(n)} \sum_{p \in \mathcal{U}_{n}} \frac{\left|\Lambda_{p}\right|^{-1}}{\left(1-\left|\Lambda_{p}\right|^{-1}\right)^{2}} w_{p}^{2} .
\end{gathered}
$$

The Hannay-Ozorio-de-Almeida uniformity sum rule $[21,29]$ implies that $\lim _{n \rightarrow \infty} g(n)=1$. Then, the limit in (15) exists only if $D_{\mathrm{WA}}(n)$ converges to $D$. For sufficiently large $n, D_{\mathrm{WA}}(n)$ in $(17)$ is just the average of $w_{p}^{2} /(2 n)\left(p \in \mathcal{U}_{n}\right)$ weighted by the stability factor $\left|\Lambda_{p}\right|^{-1}$. Such approximations to $D$ have been used in previous works $[15,18]$.

Formula (17) reduces to (4) in the case of uniform hyperbolicity. Consider, however, an equivalent expression for (17):

$$
D_{\mathrm{WA}}(n)=\frac{1}{2 n N(n)} \sum_{w} N_{w}(n) S_{w}(n) w^{2}
$$

where $N(n)$ and $N_{w}(n)$ are defined as in (4) and 


$$
S_{w}(n)=\frac{n N(n)}{g(n) N_{w}(n)} \sum_{p \in \mathcal{U}_{n}, w_{p}=w} \frac{\left|\Lambda_{p}\right|^{-1}}{\left(1-\left|\Lambda_{p}\right|^{-1}\right)^{2}} .
$$

The quantity (19) is a natural restriction of (16) to the subset of POs with given winding number. If we now assume, in analogy to $\lim _{n \rightarrow \infty} g(n)=1$, the uniformity sum rules at fixed winding number $w$,

$$
\lim _{n \rightarrow \infty} S_{w}(n)=1,
$$

formula (18) may reduce essentially to (4) also in cases of nonuniform hyperbolicity. We found much numerical evidence for the validity of (20) in our system. Part of this evidence is presented below. In general, the origin of (20) can be understood as follows. In our notation, one has the approximate relation for $n \gg 1$ (see, e.g., Appendix B in Ref. [30]):

$$
\frac{1}{\sqrt{4 \pi n D}} \exp \left(-\frac{w^{2}}{4 D n}\right) \approx n \sum_{n_{p} r=n, w_{p} r=w} \frac{\left|\Lambda_{p}^{r}\right|^{-1}}{\left(1-\left|\Lambda_{p}^{r}\right|^{-1}\right)^{2}},
$$

where $r$ (an integer) is the repetition index. The left-hand side of (21) gives the probability distribution for a generic chaotic ensemble to diffuse a "distance" $|w|$ in "time" $n$. Now, as $n \rightarrow \infty$, there should be no essential difference between such an ensemble and the PO ensemble $\mathcal{U}_{n}$. The probability distribution above is then expected to be approximately equal to $N_{w}(n) / N(n)$ provided $|w|$ is not too close to the maximal value of $\left|w_{p}\right|[10]$. We use this in (21), keeping only the dominant terms $(r=1)$ on the right-hand side. Recalling also the definition (19) and the uniformity sum rule $\lim _{n \rightarrow \infty} g(n)=1$, Rel. (20) is obtained.

In our numerical calculations we have used the relevant POs on the cylinder with periods $n \leq 14$, computed as described in Sec. II. The following PO quantities were calculated accurately for $\kappa=0.086 k, k=1, \ldots, 11$ : (a) $S_{w}(n)$ for all possible values of $w \geq 0$ [note that $S_{-w}(n)=S_{w}(n)$ from inversion symmetry]. (b) The curvature-expansion (CE) approximation to $D, D_{\mathrm{CE}}(n)$, obtained by using in (5) the expansion (7) truncated after the first $n$ terms. (c) $D_{\mathrm{WA}}(n)$ [weighted-average formula (17)]. (d) $D(n)$ [nonweighted-average formula (4)]. The PO results for $D$ were compared with standard ones obtained from (2) by choosing 
$\mathcal{E}$ as the entire unit torus. For this ensemble, which is obviously invariant under the torus map, one has the exact expansion [2]

$$
D_{\mathcal{E}}(m)=\frac{1}{2} C_{0}+\sum_{j=1}^{m-1}\left(1-\frac{j}{m}\right) C_{j}, \quad m>1,
$$

where $C_{j}=\left\langle f\left(x_{0}\right) f\left(x_{j}\right)\right\rangle_{\mathcal{E}}$ are the force-force correlations for (8). These correlations were calculated very accurately for $j \leq 30$ by a sophisticated integration of $f\left(x_{0}\right) f\left(x_{j}\right)$ over the unit torus. In general, we found that $D_{\mathcal{E}}(m)$ in $(22)$ converges rapidly to $D$ due to the fast decay of $C_{j}$. For example, $D_{\mathcal{E}}(20)$ differs from both $D_{\mathcal{E}}(10)$ and $D_{\mathcal{E}}(30)$ by no more than $0.05 \%$ for all the values of $\kappa$ considered. In what follows, $D_{\mathcal{E}}(20)$ will serve as our "standard" value $D_{\mathrm{S}}$ for $D$.

In Tables III-V we list the four quantities above for three representative values of $\kappa$; the corresponding value of $D_{\mathrm{S}}$ is also given. Table VI shows the quantities $S_{0}(14), D_{\mathrm{CE}}(14)$, $D_{\mathrm{WA}}(14), D(14)$, and $D_{\mathrm{S}}$ for all the 11 values of $\kappa$ considered. The results in Tables III-V, as well as similar results for the other values of $\kappa$, indicate that $D_{\mathrm{CE}}(n), D_{\mathrm{WA}}(n)$, and $D(n)$ start to converge, in general, for $n>8$. These quantities vanish for $n \leq 3$ since all the POs with period $n \leq 3$ have $w_{p}=0$, even for $\kappa=0.946$. Table VI shows a very good agreement between $D_{\mathrm{WA}}(14)$ and $D_{\mathrm{S}}$ for all values of $\kappa$. This agreement is generally better than that between $D_{\mathrm{CE}}(14)$ and $D_{\mathrm{S}}$. The relative difference between $D_{\mathrm{WA}}(14)$ and $D_{\mathrm{S}}$ ranges from $0.04 \%$ to $0.4 \%$ while that between $D_{\mathrm{CE}}(14)$ and $D_{\mathrm{S}}$ ranges from $0.16 \%$ to $1.7 \%$.

Table VI also shows that the relative difference between the values of $D(14)$ and $D_{\mathrm{S}}$ for $\kappa \leq 0.43$ is not larger than $2 \%$ despite the fact that the hyperbolicity for $\kappa \leq 0.43$ can be considerably nonuniform, see Fig. 2. To understand this, consider the behavior of $S_{w}(n)$ for $\kappa \leq 0.43$ in Tables III and IV. While the convergence of $S_{w}(n)$ to 1 for $|w| \leq 2$ is quite evident, $S_{3}(14)$ and $S_{4}(14)$ are not sufficiently converged due to the relatively small number of POs with $w=3,4$. Precisely because of this last fact, however, the effect of $S_{ \pm 3}(14)-1$ and $S_{ \pm 4}(14)-1$ in (18) is not significant, leading to only a small difference between $D(14)$ 
and $D_{\mathrm{WA}}(14)$ (or $\left.D_{\mathrm{S}}\right)$. As $\kappa$ is increased, a larger value of $|w|(|w|=5)$ appears for $\kappa>0.516$ and the hyperbolicity becomes more nonuniform. Then, since the total number $N(n)$ of POs is constant (does not depend on $\kappa$ ), the convergence of $S_{w}(n)$ is expected to deteriorate for all $w$. This is, in fact, confirmed by all our numerical data (see the worse case for $\kappa=0.946$ in Table V) with the exception of $w=0$. As Table VI shows, $S_{0}(14)$ remains remarkably well converged for all values of $\kappa$. We shall attempt to find an explanation of this and other facts in a future work.

\section{CONCLUSIONS}

In this paper, chaotic diffusion on periodic orbits (POs) was studied for the perturbed Arnol'd cat map on the cylinder, in a relatively large range of perturbations $\kappa$ corresponding to a "structural-stability" regime of the system on the torus. Numerical evidence indicates that this regime extends, at least approximately, significantly beyond the Anosov bound $\kappa_{\mathrm{c}} \approx 0.437$, i.e., at least up to $\kappa \approx 1$. This extension, which was already noticed in a quantum-chaos context for a different perturbed cat map [22], is further supported by the very good agreement between the PO and standard results for $D$ also for $\kappa_{\mathrm{c}}<\kappa \leq 0.946$, where only POs topologically conjugate to the $\kappa=0$ POs are used. In the absence of bifurcations, the variation of $D$ with $\kappa$ is totally due to the change of the characteristics $\left(w_{p}, \Lambda_{p}\right)$ of a constant number of POs. Thus, the case studied in this paper is basically different from that considered in Ref. [17], i.e., standard maps in a strong-chaos limit. In the latter case, bifurcations of low-period ( $n=1$ and $n=2)$ POs are the main cause for the relevant variation of $D$ with the parameter. The usual distinction between fundamental $(n=1)$ and curvature $(n>1)$ terms [19] is not felt in our case since $w_{p}=0$ for $n \leq 3$ and for all the values of $\kappa$ considered. The convergence of the PO results to $D$ generally starts only for order (or period) $n>8$.

As in the case of the Lorentz gas [18], the most accurate PO results are obtained by using 
the weighted-average formula (17). In general, by expressing this formula in the windingnumber representation (18), it becomes clear that the effect of a nonuniform hyperbolicity is completely captured by the basic quantities (19). Since these quantities satisfy the uniformity sum rules (20), the manifestation of this effect is essentially restricted, for sufficiently large $n$, to the "tail" of the distribution $(21)(|w|$ large relatively to the maximal value of $\left.\left|w_{p}\right|\right)$. Here the discrepancy between $N_{w}(n) / N(n)$ and (21) leads to a value of $S_{w}(n)$ which is not well converged. We have shown that the effect of nonconverged values of $S_{w}(n)$ may be insignificant also in cases of a considerably nonuniform hyperbolicity. Then, formula (17) reduces essentially to the nonweighted-average formula (4).

\section{Acknowledgments}

We thank J.M. Robbins for discussions. This work was partially supported by the Israel Science Foundation administered by the Israel Academy of Sciences and Humanities. V.E.C. acknowledges the CRDF and Ministry of Education of the Russian Federation for Award \#VZ-010-0. 


\section{REFERENCES}

[1] B.V. Chirikov, Phys. Rep. 52, 263 (1979), and references therein.

[2] J.R. Cary and J.D. Meiss, Phys. Rev. A 24, 2664 (1981).

[3] J.D. Meiss, J.R. Cary, C. Grebogi, J.D. Crawford, A.N. Kaufman, and H.D.I. Abarbanel, Physica (Amsterdam) 6D, 375 (1983).

[4] C.F.F. Karney, Physica (Amsterdam) 8D, 360 (1983).

[5] R.S. MacKay, J.D. Meiss, and I.C. Percival, Physica (Amsterdam) 13D, 55 (1984).

[6] I. Dana and S. Fishman, Physica (Amsterdam) 17D, 63 (1985).

[7] Y.H. Ichikawa, T. Kamimura, and T. Hatori, Physica (Amsterdam) 29D, 247 (1987).

[8] I. Dana, N.W. Murray, and I.C. Percival, Phys. Rev. Lett. 62, 233 (1989).

[9] I. Dana and M. Amit, Phys. Rev. E 51, R2731 (1995).

[10] I. Dana, Physica (Amsterdam) 39D, 205 (1989).

[11] I. Dana, Phys. Rev. Lett. 64, 2339 (1990).

[12] I. Dana and T. Kalisky, Phys. Rev. E 53, R2025 (1996).

[13] R. Artuso, Phys. Lett. A 160, 528 (1991).

[14] R. Artuso, G. Casati, and R. Lombardi, Physica (Amsterdam) 205A, 412 (1994).

[15] W.N. Vance, Phys. Rev. Lett. 69, 1356 (1992).

[16] P. Cvitanovic, P. Gaspard, and T. Schreiber, CHAOS 2, 85 (1992).

[17] B. Eckhardt, Phys. Lett. A 172, 411 (1993).

[18] G.P. Morriss and L. Rondoni, J. Stat. Phys. 75, 553 (1994).

[19] P. Cvitanovic, Physica (Amsterdam) 83D, 109 (1995), and references therein. 
[20] R. Artuso and R. Strepparava, Phys. Lett. A 236, 469 (1997).

[21] P. Gaspard, Chaos, Scattering, and Statistical Mechanics (Cambridge University Press, 1998), and references therein.

[22] M. Basilio de Matos and A.M. Ozorio de Almeida, Ann. Phys. 237, 46 (1995).

[23] P.A. Boasman and J.P. Keating, Proc. R. Soc. London A 449, 629 (1995); T.O. de Carvalho, J.P. Keating, and J.M. Robbins, J. Phys. A 31, 5631 (1998); M.V. Berry, J.P. Keating, and S.D. Prado, J. Phys. A 31, L245 (1998); J.P. Keating and F. Mezzadri, Nonlinearity 13, 747 (2000).

[24] I. Dana, Phys. Rev. Lett. 84, 5994 (2000); I. Dana, J. Phys. A: Math. Gen. 35, 3447 (2002), and references therein.

[25] V.I. Arnol'd, Geometrical Methods in the Theory of Ordinary Differential Equations (Springer-Verlag, New York, 1988).

[26] I.C. Percival and F. Vivaldi, Physica (Amsterdam) 27D, 373 (1987); Q. Chen, I. Dana, J.D. Meiss, N.W. Murray, and I.C. Percival, Physica (Amsterdam) 46D, 217 (1990).

[27] I.C. Percival and F. Vivaldi, Physica (Amsterdam) 25D, 105 (1987).

[28] J.P. Keating, Nonlinearity 4, 277 (1991); 4, 309 (1991).

[29] J.H. Hannay and A.M. Ozorio de Almeida, J. Phys. A: Math. Gen. 17, 3429 (1984).

[30] T. Dittrich, B. Mehlig, H. Schanz, and U. Smilansky, Chaos, Solitons, and Fractals 8, 1205 (1997), and references therein. 
Table I. $\zeta^{-1}(0,0)$ to order $n=1, \ldots, 14$ of curvature expansion for several values of $\kappa$.

\begin{tabular}{|r|r|r|r|r|r|r|}
\hline$n$ & \multicolumn{5}{|c|}{$\zeta^{-1}(0,0)$} \\
\cline { 2 - 6 } & $\kappa=0.086$ & $\kappa=0.258$ & $\kappa=0.43$ & $\kappa=0.602$ & $\kappa=0.774$ & $\kappa=0.946$ \\
\hline 1 & -0.36790 & -0.34306 & -0.32172 & -0.30313 & -0.28676 & -0.27220 \\
2 & -0.29773 & -0.31199 & -0.33060 & -0.35530 & -0.38853 & -0.43399 \\
3 & -0.17097 & -0.17537 & -0.17565 & -0.17293 & -0.16915 & -0.16734 \\
4 & -0.08734 & -0.09026 & -0.09120 & -0.08990 & -0.08641 & -0.08143 \\
5 & -0.04179 & -0.04339 & -0.04390 & -0.04242 & -0.03686 & -0.02308 \\
6 & -0.01918 & -0.02003 & -0.02043 & -0.01995 & -0.01786 & -0.01328 \\
7 & -0.00856 & -0.00899 & -0.00925 & -0.00909 & -0.00795 & -0.00478 \\
8 & -0.00374 & -0.00395 & -0.00410 & -0.00408 & -0.00360 & -0.00222 \\
9 & -0.00342 & -0.00688 & -0.00995 & -0.01261 & -0.01476 & -0.01621 \\
10 & -0.00002 & 0.00104 & 0.00185 & 0.00249 & 0.00306 & 0.00375 \\
11 & 0.00025 & 0.00130 & 0.00237 & 0.00350 & 0.00480 & 0.00643 \\
12 & -0.00002 & 0.00020 & 0.00042 & 0.00059 & 0.00073 & 0.00087 \\
13 & 0.00019 & 0.00061 & 0.00097 & 0.00125 & 0.00147 & 0.00164 \\
14 & 0.00012 & 0.00038 & 0.00062 & 0.00083 & 0.00099 & 0.00103 \\
\hline
\end{tabular}

Table II. Number $N(n)$ of relevant POs of period $n$, having no point on $x=-0.5$.

\begin{tabular}{|c|c|c|c|c|c|c|c|c|c|c|c|c|c|c|}
\hline$n$ & 1 & 2 & 3 & 4 & 5 & 6 & 7 & 8 & 9 & 10 & 11 & 12 & 13 & 14 \\
\hline$N(n)$ & 1 & 2 & 2 & 10 & 24 & 48 & 120 & 270 & 568 & 1500 & 3600 & 8543 & 20880 & 50700 \\
\hline
\end{tabular}


Table III. $S_{w}(n), D_{\mathrm{CE}}(n), D_{\mathrm{WA}}(n)$, and $D(n)$ for $\kappa=0.258 ; D_{\mathrm{S}}=0.04865$.

\begin{tabular}{|c|c|c|c|c|c|c|c|c|}
\hline$n$ & $S_{0}(n)$ & $S_{1}(n)$ & $S_{2}(n)$ & $S_{3}(n)$ & $S_{4}(n)$ & $D_{\mathrm{CE}}(n)$ & $D_{\mathrm{WA}}(n)$ & $D(n)$ \\
\hline 1 & 1.52221 & & & & & 0.00000 & 0.00000 & 0.00000 \\
\hline 2 & 1.19039 & & & & & 0.00000 & 0.00000 & 0.00000 \\
\hline 3 & 1.05180 & & & & & 0.00000 & 0.00000 & 0.00000 \\
\hline 4 & 1.01987 & 1.02505 & & & & 0.02724 & 0.05125 & 0.05000 \\
\hline 5 & 1.03504 & 0.97134 & & & & 0.03261 & 0.04047 & 0.04167 \\
\hline 6 & 1.02088 & 0.98225 & & & & 0.03350 & 0.03752 & 0.03819 \\
\hline 7 & 1.01632 & 0.96115 & 1.13505 & & & 0.04102 & 0.05023 & 0.04881 \\
\hline 8 & 1.02287 & 0.96467 & 1.06022 & & & 4410 & 0.04795 & 0.04768 \\
\hline 9 & 0.99896 & 0.99316 & 1.04018 & & & 0.04487 & 0.04762 & 0.04695 \\
\hline 10 & 1.01179 & 0.98401 & 1.01905 & 1.24670 & & 0.04648 & 0.04807 & 0.04760 \\
\hline 11 & 1.00966 & 0.98787 & 1.00285 & 1.17419 & & 0.04726 & 0.04862 & 0.04811 \\
\hline 12 & 1.00935 & 0.98897 & 1.00102 & 1.11421 & & 0.04751 & 0.04819 & 0.04776 \\
\hline 13 & 1.00981 & 0.98864 & 0.99965 & 1.08830 & 1.40865 & 0.04801 & 0.04873 & 0.04826 \\
\hline 14 & 1.00658 & 0.99180 & 0.99950 & 1.05561 & 1.30305 & 0.04824 & 0.04861 & 0.04815 \\
\hline
\end{tabular}


Table IV. $S_{w}(n), D_{\mathrm{CE}}(n), D_{\mathrm{WA}}(n)$, and $D(n)$ for $\kappa=0.43 ; D_{\mathrm{S}}=0.05380$.

\begin{tabular}{|c|c|c|c|c|c|c|c|c|}
\hline$n$ & $S_{0}(n)$ & $S_{1}(n)$ & $S_{2}(n)$ & $S_{3}(n)$ & $S_{4}(n)$ & $D_{\mathrm{CE}}(n)$ & $D_{\mathrm{WA}}(n)$ & $D(n)$ \\
\hline 1 & 1.47432 & & & & & 0.00000 & 0.00000 & 0.00000 \\
\hline 2 & 1.21592 & & & & & 0.00000 & 0.00000 & 0.00000 \\
\hline 3 & 1.04705 & & & & & 0.00000 & 0.00000 & 0.00000 \\
\hline 4 & 1.03876 & 0.99752 & & & & 0.02630 & 0.04988 & 0.05000 \\
\hline 5 & 1.06682 & 0.92808 & & & & 0.03128 & 0.03867 & 0.04167 \\
\hline 6 & 1.02173 & 0.95875 & 1.27251 & & & 0.03923 & 0.05429 & 0.05208 \\
\hline 7 & 1.02423 & 0.95049 & 1.13318 & & & 0.04692 & 0.05640 & 0.05476 \\
\hline 8 & 1.02965 & 0.95429 & 1.07500 & & & 0.04871 & 0.05239 & 0.05185 \\
\hline 9 & 0.99527 & 0.98363 & 1.06576 & 1.74814 & & 0.05076 & 0.05433 & 0.05184 \\
\hline 10 & 1.02227 & 0.97717 & 0.98799 & 1.35259 & & 0.05216 & 0.05339 & 0.05253 \\
\hline 11 & 1.01290 & 0.98137 & 1.00161 & 1.23369 & & 0.05265 & 0.05426 & 0.05308 \\
\hline 12 & 1.01111 & 0.98476 & 1.00131 & 1.13098 & 2.06872 & 0.05294 & 0.05318 & 0.05216 \\
\hline 13 & 1.00872 & 0.98746 & 0.99941 & 1.08594 & 1.59230 & 0.05323 & 0.05372 & 0.05271 \\
\hline 14 & 1.00869 & 0.98753 & 0.99789 & 1.07197 & 1.43866 & 0.05338 & 0.05378 & 0.05273 \\
\hline
\end{tabular}


Table V. $S_{w}(n), D_{\mathrm{CE}}(n), D_{\mathrm{WA}}(n)$, and $D(n)$ for $\kappa=0.946 ; D_{\mathrm{S}}=0.07200$.

\begin{tabular}{|c|c|c|c|c|c|c|c|c|c|}
\hline$n$ & $S_{0}(n)$ & $S_{1}(n)$ & $S_{2}(n)$ & $S_{3}(n)$ & $S_{4}(n)$ & $S_{5}(n)$ & $D_{\mathrm{CE}}(n)$ & $D_{\mathrm{WA}}(n)$ & $D(n)$ \\
\hline 1 & 1.37400 & & & & & & 0.00000 & 0.00000 & 0.00000 \\
\hline 2 & 1.42345 & & & & & & 0.00000 & 0.00000 & 0.00000 \\
\hline 3 & 1.03401 & & & & & & 0.00000 & 0.00000 & 0.00000 \\
\hline 4 & 1.19364 & 0.77294 & & & & & 0.01918 & 0.03865 & 0.05000 \\
\hline 5 & 1.05191 & 0.75048 & 2.07839 & & & & 0.05413 & 0.10055 & 0.07500 \\
\hline 6 & 1.14789 & 0.70455 & 1.63690 & & & & 0.06132 & 0.06993 & 0.06250 \\
\hline 7 & 1.06410 & 0.72943 & 1.60419 & & & & 0.06600 & 0.08195 & 0.06667 \\
\hline 8 & 1.12569 & 0.80405 & 1.16902 & 1.83040 & & & 0.06826 & 0.06781 & 0.06389 \\
\hline 9 & 0.94494 & 0.90200 & 1.33077 & 1.93640 & & & 0.07233 & 0.08259 & 0.06494 \\
\hline 10 & 1.00057 & 0.98694 & 0.91645 & 1.64065 & & & 0.07232 & 0.06972 & 0.06433 \\
\hline 11 & 0.96412 & 0.96616 & 1.09809 & 1.32642 & 1.94196 & & 0.07138 & 0.07231 & 0.06485 \\
\hline 12 & 1.01872 & 0.94809 & 1.02208 & 1.19621 & 2.92740 & & 0.07175 & 0.07120 & 0.06448 \\
\hline 13 & 0.97018 & 0.95294 & 1.10901 & 1.09196 & 2.04263 & 3.55700 & 0.07181 & 0.07262 & 0.06507 \\
\hline 14 & 1.00396 & 0.94759 & 1.04419 & 1.11549 & 1.90135 & 2.53191 & 0.07188 & 0.07180 & 0.06505 \\
\hline
\end{tabular}


Table VI. $S_{0}(14), D_{\mathrm{CE}}(14), D_{\mathrm{WA}}(14), D(14)$, and $D_{\mathrm{S}}$ for all the 11 values of $\kappa$.

\begin{tabular}{|l|l|l|l|l|l|}
\hline$\kappa$ & $S_{0}(14)$ & $D_{\mathrm{CE}}(14)$ & $D_{\mathrm{WA}}(14)$ & $D(14)$ & $D_{\mathrm{S}}$ \\
\hline 0.086 & 1.00193 & 0.04350 & 0.04377 & 0.04356 & 0.04388 \\
0.172 & 1.00431 & 0.04551 & 0.04618 & 0.04579 & 0.04622 \\
0.258 & 1.00658 & 0.04824 & 0.04861 & 0.04815 & 0.04865 \\
0.344 & 1.00752 & 0.05070 & 0.05109 & 0.05046 & 0.05116 \\
0.43 & 1.00869 & 0.05338 & 0.05378 & 0.05273 & 0.05380 \\
0.516 & 1.00807 & 0.05589 & 0.05642 & 0.05506 & 0.05656 \\
0.602 & 1.00058 & 0.05841 & 0.05938 & 0.05736 & 0.05942 \\
0.688 & 0.99577 & 0.06220 & 0.06228 & 0.05921 & 0.06240 \\
0.774 & 1.00191 & 0.06531 & 0.06548 & 0.06102 & 0.06551 \\
0.86 & 1.00014 & 0.06838 & 0.06848 & 0.06297 & 0.06873 \\
0.946 & 1.00396 & 0.07188 & 0.07180 & 0.06505 & 0.07200 \\
\hline
\end{tabular}




\section{FIGURES}

Fig. 1. Mixed phase space for the perturbed Arnol'd cat map [(1) with (8)] for $\kappa=2$.

Fig. 2. Distributions of the Lyapunov exponents $\lambda_{p}=\ln \left(\left|\Lambda_{p}\right|^{-1}\right) / n$ for all the primitive POs of period $n=14$ of the perturbed Arnol'd cat map for $\kappa=0.258$ (filled circles), $\kappa=0.43$ (crosses), and $\kappa=0.946$ (triangles). Each distribution was calculated by dividing the full range of $\lambda_{p}$ into 100 equal intervals and counting the number $\Delta N$ of values of $\lambda_{p}$ in each interval. 


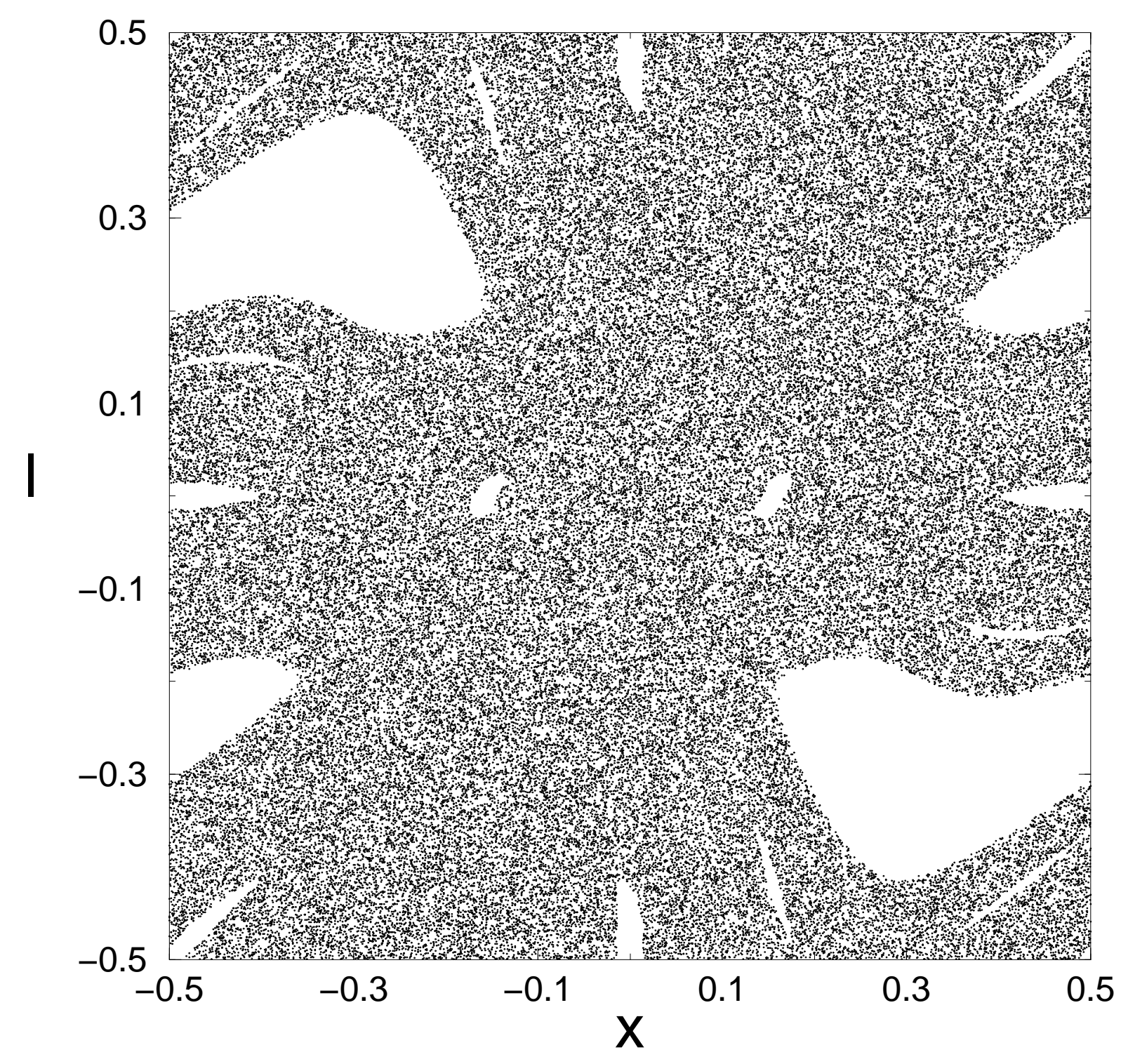




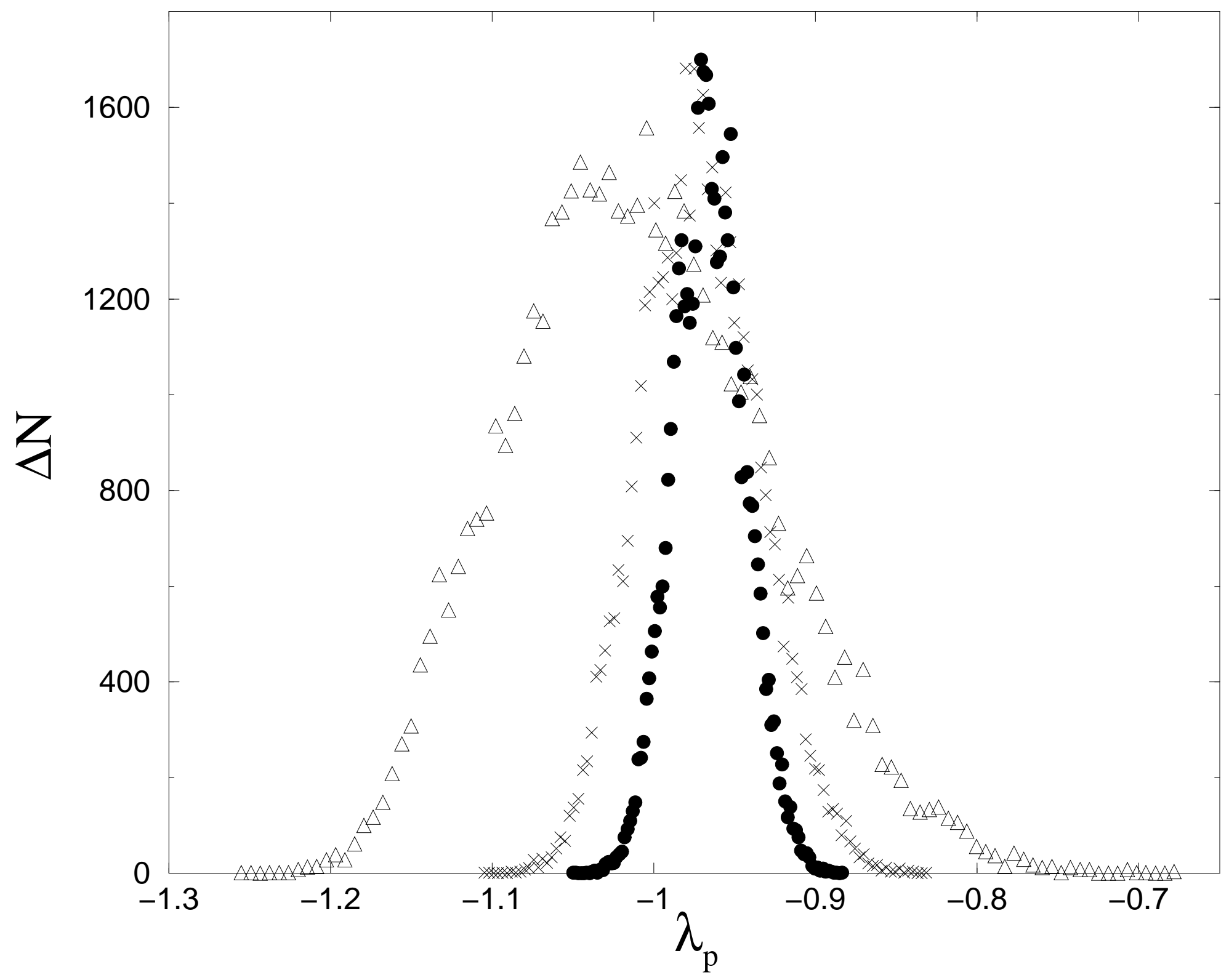

